Bull. Austral. Math. Soc.

$46 \mathrm{E} 25,30 \mathrm{D} 60$

Vol. 39 (1989) [471-476]

\title{
ON THE SUPERPOSTITION OF FUNCTIONS IN CARLEMAN CLASSES
}

\author{
Mostefa IDER
}

\begin{abstract}
In this paper we deal with classes of infinitely differentiable functions known in the literature as Carleman classes. Our main result is a characterisation of those Carleman classes that are closed under superposition. This result ensbles us to give a complete solution to a problem that has been considered by Gevrey, Cartan and Bang.
\end{abstract}

Let $M=\left\{M_{n}\right\}$ be a sequence of positive numbers and let $C_{M}(I)$ denote the Carleman class of functions $f \in C^{\infty}(I)$ which satisfy the following inequalities

$$
\left\|f^{(n)}\right\|_{\infty} \leqslant A \lambda^{n} M_{n}, n \geqslant 0, A=A(f), \lambda=\lambda(f)
$$

where $I$ is a linear interval.

A class $C_{M}(I)$ is said to be stable under superposition if for every $g \in C_{M}(J)$ and $f \in C_{M}(I)$, where $I \supset \overline{g(J)}$, the composite function $f \circ g$ is also in the class $C_{M}$. The problem of finding conditions on $M$ in order that the class $C_{M}$ be stable under superposition was first considered by Gevrey [3] for the particular Carleman classes $C_{M}$ where $M=\left\{(n !)^{\alpha}\right\}(\alpha>1)$. For the general Carleman classes Cartan [2] showed that if the sequence $A=\left\{A_{n}\right\}$ where $A_{n}=\left\{\left(M_{n} / n !\right)^{1 / n}\right\}$ is increasing then $C_{M}$ is stable under superposition. The question as to whether the converse of this result is true remains unsolved. However if we suppose that the class $C_{M}$ is differentiable, in the sense that $f \in C_{M}$ implies $f^{\prime} \in C_{M}$, we are able to give a complete solution to this problem by showing that in this case a condition weaker than that of Cartan is both necessary and sufficient. We recall that the class $C_{M}(I)$ is inverse-closed if for every $f \in C_{M}(I)$ such that $f(x) \neq 0, f^{-1} \in C_{M}(I)$. It was Malliavin [4] who first gave a sufficient condition in order that $C_{M}(I)$ be inverse-closed, by showing that if the sequence $A$ is almost increasing, that is $\left(\exists K>0\right.$, s.t $\left.\forall n \leqslant m, A_{n} \leqslant K A_{m}\right)$, then the class $C_{M}$ is inverseclosed. Rudin [6] proved that if $C_{M}$ is a non-quasianalytic class of $2 \pi$-periodic functions then the converse of Malliavin's result is also true. To avoid the trivial cases we will suppose that $\lim _{n \rightarrow \infty} M_{n}^{1 / n}=\infty$ and hence the class $C_{M}(\mathbf{R}) \equiv C_{M^{c}}(\mathbf{R})$ where $\left\{\log M_{n}^{c}\right\}$ is the largest convex minorant of $\left\{\log M_{n}\right\}$ (see Madelbrojt [5]). If $\underset{n \rightarrow \infty}{\lim _{n}} M_{n}^{1 / n}=0$,

Received 23 August 1988

Copyright Clearance Centre, Inc. Serial-fee code: 0004-9729/89 \$A2.00+0.00. 
then $C_{M}(\mathbf{R})$ is reduced to the class of constant functions (see Madelbrojt [5]) which is obviously inverse-closed and if $\underline{\lim }_{n \rightarrow \infty} M_{n}^{1 / n}<+\infty$, then $C_{M}(\mathbf{R}) \equiv C_{\{1\}}(\mathbf{R})$ which is not inverse-closed, since if $f(x)=\sin x, f \in C_{\{1\}}(\mathbf{R})$ but $f \circ f \notin C_{\{1\}}(\mathbf{R})$ (see Bang [1]). Without loss of generality we will therefore suppose that $M=M^{\mathrm{c}}$. Using the techniques developed in [7] we are now in the position to prove the following

THEOREM 1. The following assertions are equivalent

(i) the sequence $A$ is almost increasing;

(ii) if $f \in C_{M}(R)$ and $f$ is analytic in a domain containing the closure of the range of $g$ then $f \circ g \in C_{M}(\mathbf{R})$;

(iii) $C_{M}(\mathbf{R})$ is inverse-closed.

Proof: That (i) implies (ii) follows directly from the formula of Faà Di Bruno, namely

$$
(f \circ g)^{(n)}(x)=\sum \frac{n !}{k_{1} ! k_{2} ! \ldots k_{n}} f^{(k)}[g(x)]\left(\frac{g^{\prime}(x)}{1 !}\right)^{k_{1}} \ldots\left(\frac{g^{(n)}(x)}{n !}\right)^{k_{n}}
$$

where the summation is over all the $n$-tuples $\left(k_{1}, k_{2}, \ldots, k_{n}\right)$ such that $k_{1}+k_{2}+\ldots+$ $k_{n}=k$ and $k_{1}+2 k_{2}+\ldots+n k_{n}=n$.

Trivially (ii) implies (iii). We now show that (iii) implies (i). Let

$$
g(x)=\sum_{v=1}^{\infty} \frac{1}{2^{v}} \frac{e^{i k_{v} x}}{T_{M}\left(k_{v}\right)}
$$

where (see Madelbrojt [5])

$$
T_{M}(r)=\sup _{n>0} \frac{r^{n}}{M_{n}}
$$

and

$$
M_{n}=\sup _{r>0} \frac{r^{n}}{T_{M}(r)}
$$

It can be easily seen that

$$
\forall x \in \mathbf{R} \quad\left|g^{(n)}(x)\right| \leqslant M_{n}, n \geqslant 0
$$

and that

$$
g^{(n)}(0)=i^{n} s_{n}
$$

where

$$
s_{n} \geqslant \frac{1}{2^{n}} M_{n}
$$


Choose $f(x)=1 /(\lambda-x)$, where $\lambda>M_{0}$. Since $\lambda-g \in C_{M}(\mathbf{R})$ and $C_{M}(\mathbf{R})$ is inverse closed, it follows that $(f \circ g)=(\lambda-g)^{-1} \in C_{M}(\mathbf{R})$. Now choosing $k_{s}=k, k_{j}=0$ for $j \neq s, n=k s$ in the formula (1) applied to the composite function $f \circ g$ at the point $x=0$ we get

$$
\left(\frac{M_{s}}{s !}\right)^{1 / 0} \leqslant K\left(\frac{M_{n}}{n !}\right)^{1 / n}
$$

If $n$ is not a multiple of $s$, let $s m<n<s(m+1)$ and so using the fact that $\left\{M_{n}^{1 / n}\right\}$ is increasing we obtain

$$
\frac{M_{n}^{1 / n}}{n} \geqslant \frac{M_{s m}^{1 / s m}}{s(m+1)} \geqslant \frac{M_{s m}^{1 / s m}}{s m} \cdot \frac{s m}{s(m+1)} \geqslant \frac{1}{2} \frac{M_{s}^{1 / s}}{s} .
$$

Thus for $s \leqslant n$

$$
\left(\frac{M_{s}}{s !}\right)^{1 / 0} \leqslant K\left(\frac{M_{n}}{n !}\right)^{1 / n}
$$

where $K$ is independent of $s$ and $n$, that is $A$ is almost increasing.

As remarked earlier Cartan [2] has shown that if $A$ is increasing then the class $C_{M}$ is stable under superposition. We now show that this result remains true if we suppose more generally that the sequence $A^{\prime}=\left\{\left(M_{n} / n !\right)^{1 / n-1}\right\}$ is almost increasing. In fact we have the following

THEOREM 2. If the sequence $A^{\prime}$ is almost increasing then the class $C_{M}(I)$ is stable under superposition.

Proof: Let $g \in C_{M}(J)$ and $f \in C_{M}(I)$, where $I \supset \overline{g(J)}$, then applying formula (1) we get

$$
\left|(f \circ g)^{(n)}(x)\right| \leqslant C \mu^{n} \sum \frac{n !}{k_{1} ! \ldots k_{n} !} M_{k}\left(\frac{M_{1}}{1 !}\right)^{k_{1}} \ldots\left(\frac{M_{n}}{n !}\right)^{k_{n}} .
$$

But, since $A^{\prime}$ is almost increasing, we have

$$
\left(\frac{M_{2}}{2 !}\right)^{k_{2}} \cdots\left(\frac{M_{n}}{n !}\right)^{k_{n}} \leqslant K^{n-k}\left(\frac{M_{n}}{n !}\right)^{\left(k_{2}+2 k_{3}+\ldots+(n-1) k_{n}\right) /(n-1)}
$$

and

$$
M_{k} \leqslant k ! K^{-k-1}\left(\frac{M_{n}}{n !}\right)^{(k-1) /(n-1)} .
$$

And so from (3) it follows that

$$
\left|(f \circ g)^{(n)}(x)\right| \leqslant B_{1} \lambda^{n} M_{n} \sum \frac{k !}{k_{1} ! \ldots k_{n} !}
$$


Now using the identity

$$
\sum \frac{k !}{k_{1} ! \ldots k_{n} !}=2^{n-1}
$$

we obtain

$$
\left|(f \circ g)^{(n)}(x)\right| \leqslant B \lambda^{n} M_{n}
$$

We now prove that the converse of Theorem 2 is true if the class $C_{M}(\mathbf{R})$ is differentiable. In face we prove the following

TheOREM 3. The following assertions are equivalent

(i) the sequence $A$ is almost increasing;

(ii) the class $C_{M}(\mathbf{R})$ is stable under superposition, provided that $A$ is not bounded.

ProOF: (i) implies (ii). If $A$ is almost increasing, then

$$
\forall s \leqslant n \quad\left(\frac{M_{s}}{s !}\right)^{1 / s} \leqslant K\left(\frac{M_{n}}{n !}\right)^{1 / s},
$$

and so

$$
\left(\frac{M_{s}}{s !}\right)^{1 / s-1} \leqslant K\left(\frac{M_{n}}{n !}\right)^{1 / n-1} \cdot\left(\frac{M_{s}}{s !}\right)^{1 / n(s-1)} .
$$

But since the class $C_{M}(R)$ is differentiable, we have

$$
\left(\frac{M_{s}}{s !}\right)^{1 / n(s-1)} \leqslant K_{1}
$$

and so the sequence $\left\{\left(M_{n} / n !\right)^{1 / n-1}\right\}$ is almost increasing. Now by Theorem 2 , we get that $C_{M}(R)$ is stable under superposition. Thus (i) implies (ii). Conversely, if $C_{M}(\mathbf{R})$ is stable under superposition and $A$ is not bounded, then $C_{M}(\mathbf{R})$ is stable under composition with analytic functions and so it is inverse-closed. It follows by Theorem 1 that the sequence $\left\{\left(M_{n} / n !\right)^{1 / n}\right\}$ is almost increasing. Thus (ii) implied (i). Now, someone may ask if it is possible to find a Carleman class for which our result applies but Cartan's does not, and the answer is positive. Let $M=\left\{M_{n}\right\}$ be the following sequence

$$
M_{n}^{1 / n}=n+1
$$

Then it is clear that $M$ is log-convex, the class $C_{M}(\mathbf{R})$ is differentiable, the associated sequence $A=\left(M_{n} / n !\right)^{1 / n}$ is almost increasing but not increasing.

We now show that if the sequence $A$ is bounded, then, in general, $C_{M}(\boldsymbol{R})$ is not stable under superposition. In fact we have the following result. 
THEOREM 4. If the sequence $A$ is decreasing to 0 , then there exist two functions $g \in C_{M}(\mathbf{R})$ and $f \in C_{M}(J)$ where $J \supset \overline{g(\mathbf{R})}$ such that $f \circ g \notin C_{M}$.

Proof: Since the sequence $A$ is decreasing to 0 , this enables us to construct a function $f$ belonging to $C_{M}(\mathbf{R})$ such that $f^{(n)}(0)=M_{n}$, for all $n \geqslant 0$. In fact since $A$ is decreasing, then there exists a constant $K$ such that

$$
\forall s \leqslant n \quad \frac{M_{n}}{n !} \leqslant K^{n+1} \frac{M_{0}}{s !}
$$

Puting $s=0$ in (4) we get

$$
M_{n} \leqslant K^{n+1} n !
$$

and so it follows that the series

$$
f(x)=\sum_{n=0}^{\infty} \frac{M_{n}}{n !} x^{n}
$$

converges for $|x|<1 / K$, and hence $f^{(k)}(0)=M_{k}$. We have also

$$
\left|f^{(k)}(x)\right| \leqslant \sum_{n=0}^{\infty}\left|\frac{x^{n-k} M_{n}}{(n-k)}\right| \leqslant \sum_{n=0}^{\infty}\left|x^{n-k}\right| K^{n+1}\left(\begin{array}{l}
n \\
k
\end{array}\right) M_{k} .
$$

But since $\left(\begin{array}{l}n \\ k\end{array}\right) \leqslant 2^{n}$ we have

$$
\left|f^{(k)}(x)\right| \leqslant A \lambda^{k} M_{k}
$$

Thus $f$ belongs to $C_{M}(\mathbf{R})$ in a neighbourhood of the origin. But since $C_{M}(\mathbf{R})$ is analytic, $f \in C_{M}(\mathbf{R})$. Choose now

$$
g(x)=\sum_{v=1}^{\infty} \frac{e^{i k_{v} x}}{T_{M}\left(k_{v}\right)} .
$$

We have already shown that $g \in C_{M}(\mathbf{R})$ and that

$$
g^{(n)}(0)=i^{n} s_{n}
$$

where

$$
s_{n} \geqslant \frac{1}{2^{n}} M_{n}
$$

Suppose now that the composite function $f \circ g \in C_{M}(\mathbf{R})$. Then applying formula (1) to $f \circ g$ at the point $x=0$ and $\forall j \neq s$, choosing $k_{s}=k, k_{j}=0$ where $n=k s$ we have

$$
\frac{n ! M_{k}}{2^{n} k !}\left(\frac{M_{s}}{s !}\right)^{k} \leqslant B \mu^{n} M_{n}
$$


Thus

$$
\left(\frac{M_{k}}{k !}\right)\left(\frac{M_{s}}{s !}\right)^{k} \leqslant B 2 \mu^{n}\left(\frac{M_{n}}{n !}\right) .
$$

Now, using the fact that the sequence $A$ is decreasing, and taking the $n$th root of both sides, we get

$$
\left(\frac{M_{s}}{s !}\right)^{1 / \bullet} \leqslant B^{1 / k s} 2 \mu\left(\frac{M_{n}}{n !}\right)^{(1 / k s) s-(1 / 0)} .
$$

If now we let $k$ tend to infinity we have

$$
M_{s}=0, \forall s \geqslant 0
$$

that is the class $C_{M}(\mathrm{R})$ is trivial, which is not the case and so $f \circ g \notin C_{M}(\mathbf{R})$. If $n$ is not a multiple of $s$ we proceed as in Theorem 1 and the proof of the Theorem is complete.

\section{REFERENCES}

[1] T. Bang, On quasi-analytisk funktioner (Nyt Norclisk Forlag, Copenhagen, 1946).

[2] H. Cartan, Sur les classes de fonctions définies par des inégalités sur leurs dérivées successives (Actualités Scientifiques et industrielles numéro 897, 1940).

[3] M. Gevrey, Oeuvres complètes (Editions du Centre National de la recherche scientifique, Paris, 1970).

[4] P. Malliavin, 'Calcul symbolique et sous algèbres de $L^{1}(G)$ ', Bull. Soc. Math. France 87 (1959), 181-190.

[5] S. Mandelbrojt, Series adhérentes, régularisation des suites, applications (Gauthier-Villars, Paris, 1952).

[6] W. Rudin, 'Division in algebras of infinitely differentiable functions', J. Math. Mech 11 (1962), 797-809.

[7] J.A. Siddiqi and M. Ider, 'Symbolic Calculus in analytic Carleman classes', Proc. Amer. Math. Soc. 99 (1987), 347-350.

Département de mathématiques et d'informatique Université de Sherbrooke

Sherbrooke Québec

Canada JIK 2R1 